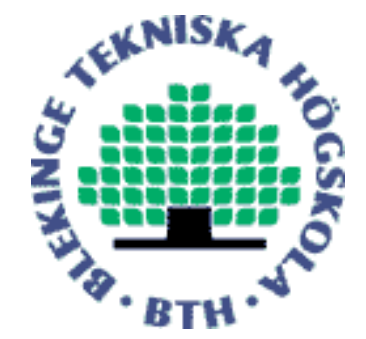

Copyright (C) 2012 IEEE.

Citation for the published paper:

Beacon-Enabled Cognitive Access for Dynamic Spectrum Access

Said Rutabayiro Ngoga, Yong Yao, Adrian Popescu

8th Euro-NF Conference on Next Generation Internet (NGI)

2012 Karlskrona

This material is posted here with permission of the IEEE. Such permission of the IEEE does not in any way imply IEEE endorsement of any of BTH's products or services Internal or personal use of this material is permitted. However, permission to reprint/republish this material for advertising or promotional purposes or for creating new collective works for resale or redistribution must be obtained from the IEEE by sending a blank email message to pubs-permissions@ieee.org.

By choosing to view this document, you agree to all provisions of the copyright laws protecting it. 


\title{
Beacon-Enabled Cognitive Access for Dynamic Spectrum Access
}

\author{
Said Rutabayiro Ngoga, Yong Yao and Adrian Popescu \\ School of Computing, \\ Blekinge Institute of Technology, \\ 37179 Karlskrona, Sweden, \\ Email: \{srn,yya,apo\}@bth.se
}

\begin{abstract}
In dynamic spectrum access networks, the unused licensed spectrum used by primary users (PU) is opened to unlicensed secondary users (SU) for improving spectrum efficiency. We design a simple time-based threshold policy for collective protection of PUs, enabled by an out-of-band channel. In particular, multiple SUs may be widely distributed in a geographic location. The interference that collocated SUs cause to each other, termed self-interference, becomes a major source that may degrade the SUs communication performance. We establish an analytical framework for carrier sense multiple access (CSMA) based coexistence mechanisms when integrated into a family of time based threshold policies, and study its performance though theoretical analysis.
\end{abstract}

\section{INTRODUCTION}

The dramatic increase of wireless services and applications is severely limited by the scarcity of the resources like bandwidth and energy. At the same time, measurements made on spectrum usage indicate that a large portion of licensed spectrum is unused at any given time and location [14]. This confirms the availability of many spectrum opportunities along time, frequency and space axes.

The inefficient usage of spectrum, thus, advocates for the development of dynamic spectrum access (DSA) techniques. This allows unlicensed users, known as secondary users (SUs), to use the temporarily unused spectrum of licensed users, also known as (PUs), through the use of cognitive radio (CR) technology [10].

A SU may not be able to simultaneously sense all channels in the spectrum, due to hardware and energy cost limitations. Under the PU protection requirements and the dynamics in the spectrum availability, characteristics of opportunistic spectrum access (OSA) model, the SU must have an efficient channel selection and access strategy in order to maximize its throughput. In particular, the access strategy includes both sensing and transmission strategies, which should be jointly designed to satisfy the PU protection requirement. Efficient sensing and transmission methods for slotted PU and SU were proposed in [15] allowing SU to search for spectrum opportunities without a central coordinator. The slotted structure simplifies the problem such that the work in [1] establishes a separation principle that decouples sensing from transmitting. The work in [15] adopts a different approach to cognitive access, where the PU transmission structure is unslotted and the PU activity follows a continuous-time Markov model. When SU overlays the unslotted PU, regardless of perfect sensing or not, a SU can not detect a PU during transmission, which further results in collision with PU. To obtain the optimal access strategy, the authors restrict the sensing strategy to a periodic scheme where the SU periodically senses the available channel. The periodic sensing structure on SU allows the auther of [8] to develop on the performance analysis of the optimal access strategy in which lower and upper bounds on performance are determined. A generalization on the PU traffic patterns is addressed in [13],[4], in which the authors establishe the time capacity of SU access.

In this paper, we focus on the hierarchical access model of DSA, also referred to as opportunistic spectrum access (OSA). In this model, the licensed spectrum of PUs is opened to SUs under the protection requirement on PU's activity [12]. The activity of PU consequently changes the availability of a spectrum band. In this case, the OSA model allows SU to search for, identify, and exploit the idle periods of PU while protecting PU's activity. In specific, SUs seek opportunities for transmission by exploiting the idle periods left between PU's packets transmission while protecting PU activities.

The medium access control (MAC) protocols coordinate communication over wireless channels and resolve channel access contentions. For OSA networks, the MAC protocols of SUs should be able to achieve cognitive access such that the negative effect of SUs on the PU activities is limited. The problem becomes more challenging when there are multiple decentralized SUs, or collocated SUs, attempting to access a common PU channel. This is because the SUs may be widely distributed such that one SU may not be able to sense all other SU's transmissions. This problem is known as the hidden problem. In this case, the interference that collocated SUs may cause to each other, termed self-interference, becomes a major source that may degrade SUs communication performance. Efficient coexistence mechanisms among collocated SUs are therefore needed to be integrated into the cognitive access to address the self-interference problem. By this, a cognitive MAC is able to negotiate and to make reservation of the common available channel before transmission initiation while guaranteeing PU performance requirement.

Referred to as threshold policy, in which the time capacity for SU cannot exceed the product of collision probability constraint and the idle percentage of the PU's traffic, the goal 
of this paper is to design distributed cognitive access based on a family of threshold policies. This is enabled by an out-ofband channel termed global common communication channel (CCC). Specifically, we study CSMA based coexistence mechanisms when integrated into a family of time based threshold policies. A distinguished feature of our paper is that we analyze CSMA based coexistence mechanisms for collocated SUs while explicitly taking into account the interference to the PU. Specifically, the SUs should search for transmission opportunities on the PU channel, coordinate among themselves to share available opportunities and transmit within the given collision constraint.

We consider a system setup with one PU following a channel occupancy pattern model together with a set of collocated SUs. We model the spectrum usage patterns of PUs as independent continuous time ON-OFF Markov processes with "OFF" indicating a channel free from PU activities and "ON" indicating the channel being used by PU. This model is a reasonable approximation of existing wireless access applications ftp, http, and VoIP [2]. We adopt packet collision probability as the PU protection requirement. Typically, the collective activity of SUs must guarantee that the packet collision probability of a PU packet is less than a certain threshold a priori specified by PU.

Referred to as beacon-enabled threshold policy, the SUs enter an active period followed by a vacation period when the channel is sensed idle during a beacon interval (BI). If the channel is sensed busy, the SUs keep sensing until the channel become idle. The family of threshold policies were first studied in [4] and [3] with the goal to study the time capacity of SUs under the PU protection requirement. In addition, CSMA based protocols have been widely used with the goal to maximize system throughput when multiple users contend for channel access. In comparison, here we develop a family of CSMA access schemes specific for collocated SUs enabling each SU to transmits less aggressively to accommodate other SUs when sharing a common available PU channel. Furthermore, we suggest a random access based coexistence mechanism implemented using the time-threshold policies and study its performance through a theoretical analysis.

The rest of the paper is organized as follow. Section II describes the system model that characterizes both PU and SU activities. Section III describes the channel access policy allowing SU to access PU channel. Section IV presents the channel negotiation and reservation protocol for the common available channel. Section V introduces the system parameters and performance analysis. Section VI presents numerical results. Section VI concludes the paper.

\section{System Model}

We consider a single spectrum band that is assigned to the PU. A set of collocated SUs exploit spectrum opportunities left by the PU under the protection requirements of the PU.

\section{A. PU Model}

The traffic statistics of PU are such that the channel occupancy by PU evolves independently according to an alternating IDLE(0)-BUSY(1) process, which is a reasonable approximation of existing wireless access applications (ftp, http, and VoIP) [2],[15]. We consider a packet-like system for the PU such that PU's packets are transmitted during some busy period. After all PU packets, including the last packet in the queue, have been transmitted, the PU channel becomes idle. The channel remains idle for a period until the next PU packet arrives, in which case a new busy-idle cycle starts.

The idle periods are considered to be exponentially distributed with known parameter denoted $\lambda^{-1}$. Similarly, the busy periods period are exponentially distributed with parameter denoted $\mu^{-1}$. The stationary distribution associated with the PU activities can thus be described by:

$$
\rho(0)=\frac{\mu}{\lambda+\mu} \quad \rho(1)=\frac{\lambda}{\lambda+\mu}
$$

\section{B. SU Model}

We assume a packet-like and time-slotted system for the SUs. To keep SUs node simple, a single transceiver per node is assumed to carry out sensing and transmission functions. In particular, we consider a single hop network in which a set of collocated SUs form a distributed CR network. All SUs are in close proximity to each other and communicate in peer-to-peer fashion. Such scenarios are of practical appeal because practical deployment may not always allow to set up a centralized controller that coordinates the activities of SUs. Instead, we assume there is a dedicated global channel control channel (CCC) to facilitate the exchange of control messages. At the MAC layer, an out-of-band requirement on a single transceiver of SU means to interrupt data transmissions for some period to carry out the channel negotiation and reservation function.

Similar to [5],[6] we assume that the network operates in beacon enabled mode. This allows a synchronous operation of the network so that SUs can enter active and vacation modes at designed time slots. Specifically, the time is divided into beacon intervals (BIs). We assume that each SU in the network is synchronized by periodic beacon transmissions, so that every SU starts and finishes each beacon interval almost at the same time. Whenever a SU has a packet to transmit it needs to join the network. A SU with data to transmit listens to the beacon signal on the CCC to synchronize itself with the rest of the network. In the case it does not hear any beacon signal, it is assumed that it is the first node in the network and thus starts sending periodic beacon signals.

Furthermore, each BI includes an active period (transmission) followed by a vacation period where SUs are not allowed to transmit. The active period is composed of three phases: sensing, contention and data transmission. In the following we describe how the SUs interact during the three phases.

1) Sensing: the active period starts with the transmission of a beacon signal and sensing begins immediately after the beacon. During sensing, SUs are not allowed to transmit hence 
the sensing result are error-free among SUs. Instead, each SU senses the PU channel independently and maintains the PU activities status by the end of the sensing period.

2) Contention: immediately after the sensing phase, if the channel is deemed idle, every SU with data to transmit enters a contention phase. Every SU of the network participates in the channel negotiation and reservation on the CCC by using some slotted CSMA/CA mechanisms extended with the Ready-ToSend(RTS)/Clear-To-Send(CTS) mode.

In specific, a SU sender reserves the channel for data transmission by sending an RTS (Ready to Send) packet to its intended SU receiver. The SU receiver replies upon receiving RTS, by sending CTS (clear to send) packet to the sender. RTS and CTS packets include both the expected duration of time for which the channel is reserved for use and the identity of the common available PU channel. In addition, the RTS/CTS messages allow collocated SUs around the sender and the receiver to avoid transmitting on a reserved channel, thus avoiding the hidden terminal problem. Other SUs of the network that overhear these messages set a NAV (network allocation vector) for the reserved channel. Hence, the other SUs must defer their transmission until the next beacon time.

3) Data transmission: after the contention phase, the SU that has successfully reserved the common available channel starts transmission, which in turn ends the active period.

\section{Channel ACCess Policies}

Regardless of the perfect sensing of SUs, collision to PU can still occur in the data transmission phase. Though the channel reservation mechanism can significantly reduce the impact of SUs transmissions on the PU's activities, this can accumulate to a degree that is unacceptable to PU. Thus, one of the main objectives is to design distributed access schemes to achieve the collective protection to PU. In this section we describe the collective access policies allowing SUs to access the PU channel under the PU protection requirement.

\section{A. SU Cognitive Access Policies}

We know that a cognitive cycle is bounded by beacon signals and it includes an active period followed by a vacation period, which can be adjusted such as the protection to PU is met. During the active period all SUs stay awake and only one transmission (successful SU transmission or SU collision) can take place. In the vacation period all SUs enter sleep mode to save energy. The cognitive cycle starts with beacon signal, and the collective sensing starts after the beacon. Afterwards, the channel status is assumed to be known at the end of the sensing period. For simplicity, we assume that SU performs perfect sensing on PU channel [15],[13],[4]. That is, the collective sensing and beacon intervals are negligible and the sensing outcomes are error-free. If the channel is sensed busy, SUs are not allowed to transmit and they will sense the channel again in the subsequent cognitive cycle. If the channel is deemed idle, a transmission (successful SU transmission or self collision) will take place during the active period followed by a vacation period. In case a transmission from a tagged
SU takes place and PU channel remains idle for the entire duration of transmission of the tagged SU, the SUs network earns a unit reward for non-intrusive access. Otherwise, if the PU returns in the middle of the SU transmission period, a PU packet collision happens in which case the SUs violate the PU protection requirement.

\section{B. PU Packet Collision Probability}

Following the above algorithm, there is at most one PU packet experiencing collision within a busy-idle cycle. Under the assumption of perfect sensing, this can only occur at the beginning of a busy period when the PU activities resume. If the current cognitive cycle ends with collision in the subsequent cognitive cycle, to avoid colliding more PU packets the SUs will refrain from transmitting until the channel becomes idle.

Similar to [13],[4] the PU has a packet probability collision requirement that we denote as $\eta$. This is defined as the maximum probability of collision for a PU's packet that PU can tolerate. Over a long run time interval $T$, we denote $N$ as the number of packets transmitted by PU and $N_{c}$ as the number of PU packets experiencing collision. Therefore, the collision probability associated with the SUs access policy is denoted as $p_{c}$, where

$$
p_{c}=\lim _{T \rightarrow \infty} \frac{N_{c}}{N}
$$

such that the PU protection requirement is

$$
p_{c} \leq \eta
$$

which is also the collision constraint imposed on the collective access of SUs.

\section{Channel Negotiation And Reservation}

So far, the structure of the cognitive cycle has been defined. Here, we emphasize that the contention phase is a small price to pay in an environment with high dynamics in spectrum usage. Thus, the contention overhead needs particular attention in designing distributed channel access schemes that allow SUs to operate under PU protection requirement.

First, we identify the various events that arise when SUs interact in attempt to access the PU channel. Then, from an individual SU viewpoint referred to as a tagged node, we derive the analytical model, the parameters of which will be described in Sec. V. This directly relates the MAC performance metrics such as SU throughput and delay.

\section{A. CSMA-like Based Random Access}

During the contention phase, the exchange of control messages by SUs takes place on CCC using the CSMA-like mechanisms. A cognitive cycle is divided into equal size time slots of fixed unit length called backoff slot. Starting from the first slot the channel is deemed idle, a contending SU with data to transmit starts with a random backoff. The backoff duration is randomly chosen in the range $\left[0, B_{0}-1\right]$, where $B_{0}$ is the initial backoff window at stage 0 . The backoff 
counter decrements by one in the subsequent slots. Once the backoff period is completed (i.e., reaches 0), the contending SU seeks a reservation of the channel. It samples the channel to see if the channel is free, in which case the channel will be reserved for transmission. Otherwise, the channel is not free and the contending SU starts a second random backoff in the range $\left[0, B_{1}\right]$ where $B_{1}$ is the backoff window at stage 1 , typically with $B_{1}>B_{0}$. For all neighboring SUs, the counter is frozen when a transmission starts until the subsequent cognitive cycle. Each SU that has successfully reserved the PU channel transmits the packet. Notice that this may result either in a successful transmission or in a self-collision (i.e., collision among SUs).

\section{B. Stochastic Model for the Backoff Procedure}

Following the CSMA family of protocols for channel negotiation and reservation, a common characteristic is that every SU of the network individually implements a backoff procedure to access the channel. The backoff procedure is often interrupted by transmissions from neighborhoods and resumes after the channel is deemed idle. This naturally forms a regenerative process having the property that there exist time points called renewal points at which the process restarts itself.

Let $B_{i}, i=0,1, \ldots,(M-1)$ be the backoff window at stage $i$, where $M$ denotes the maximum number of stages. We define the $X$-renewal cycle as the duration between contiguous time instants where a tagged SU starts a stage 0 backoff. This is the period from the time a tagged node finishes a transmission until the end of the next one. The number of access attempts, denoted $R$, carried out by the tagged SU, is regarded as a reward associated with the $X$-cycle. Notice that a $X$-cycle associated with a tagged SU can either include no transmission or a SU transmission. If the number of backoff stage exceeds $M$, the SU packet is discarded, in which case the $X$-cycle is of type $X_{1}$, denoted $X_{1}$-cycle. Otherwise, the $X$-cycle is of type $X_{2}$, denoted $X_{2}$-cycle, when it includes a SU transmission, in which case we assume the SU transmission ends this particular $X$-cycle.

Over a large time scale, we can also set the renewal points denoted $Y$-cycle at the end of each $X_{2}$ cycle including SU transmission. We assume that a successful transmission is always accompanied by ACK (MAC acknowledgement). Depending of the result of transmission, i.e., the ACK value, a $Y$-cycle can be of type $Y_{1}$, denoted $Y_{1}$-cycle, in case the transmission ends in self collision. In the case a successful transmission takes place, the $Y$-cycle is of type $Y_{2}$, denoted $Y_{2}$-cycle. In this case we assume a successful SU transmission ends the $Y$-cycle.

Regarding the MAC performance, for simplicity purposes all idle channel time is assumed to correspond to the contention phase of the cognitive cycle. Similar to [9], the backoff procedure implemented during the contention phase, especially the backoff counter decrement, occurs only in this phase and freezes during the transmission phase and vacation period. The impact of both the transmission and vacation period on the
MAC performance are then taken as having constant time cost associated to every contention slots. .

\section{SU MAC Performance Metrics}

Define a discrete random variable $v$, as the number of $X$-cycle contained in the $Y$-cycle, to be the number of transmission attempts up to and including the first transmission from a tagged SU. Clearly, $v$ is geometrically distributed with parameter $P_{v}$, which is the probability that a $X$ - cycle contains a transmission. It follows that

$$
E[Y]=\frac{E[X]}{P_{v}}
$$

The SU MAC performance metrics are as follows:

1) SU Packet Delay: let $D_{s}$, referred to as the service time of a SU packet, be the average duration from the instant a SU packet becomes the head-of-line at the SU MAC until it is successfully transmitted. Define a discrete random variable $\omega$, as the number of $Y$-cycle at steady state, to be the number of transmissions conducted by the tagged SU up to and including a successful transmission. Clearly, $\omega$ is geometrically distributed with parameter $P_{\omega}$, which is the probability that a $X$-cycle achieves a successful transmission. It follows that

$$
D_{s}=\frac{E[Y]}{P_{\omega}}
$$

2) SU Throughput: if the SU transmission lasts $K$ slots, the MAC throughput of an individual $\mathrm{SU}$ is given by

$$
\Gamma_{s}=\frac{K}{D_{s}}
$$

while the aggregated throughput for a number of $N$ collocated SUs is given by

$$
\Gamma=N \Gamma_{s}
$$

\section{System Parameters And Performance Analysis}

Prior to obtaining the performance results for the SUs channel access policy, we introduce several important parameters of the system model.

\section{A. Derivation of $\beta$}

Assume a homogeneous SUs network, which means that all SUs use the same backoff parameters. More specifically, the backoff parameters are defined as follow:

$$
\begin{aligned}
M:= & \text { At the } M \text { th backoff stage, either the SU packet } \\
& \text { is discarded or transmitted. } \\
b_{m}:= & \text { The mean backoff duration (in unit backoff slots) } \\
& \text { at stage } m \\
= & 2^{m} \times 2^{(h-1)} \quad m=0,1,2, \ldots, M \text { and } h>0 \\
K:= & \text { SU packet duration (in unit backoff slots) } \\
N:= & \text { Number of collocated SUs }
\end{aligned}
$$

Following this, $\beta$ denotes the rate at which every SUs of the network attempts a channel reservation, i.e.,

$$
\beta:=\operatorname{Pr}(\text { attempts to make a channel reservation) }
$$


By the decoupling approximation [11],[7], we assume that all SUs achieve the same value of $\beta$. Suppose there are $N$ collocated SUs. From the view point of a tagged SU, the "influence" of the rest $(N-1)$ SUs is modeled such that the aggregated backoff process of the $(N-1)$ is independent of the backoff process of the tagged SU. This allows us to encapsulate the effect of rest of collocated SUs on the tagged SU into one parameter, i.e., $\beta$. In specific, we do no track when all other SUs are in their respective states, whether transmit or backoff.

Let $\alpha$ denote the probability of channel reservation failure by a tagged SU, i.e.,

$\alpha:=\operatorname{Pr}($ a channel reservation attempt by a tagged SU fails),

which is defined as the probability of finding a channel occupied when a tagged SU completes its backoff. It follows that, with probability $(1-\alpha)$, the channel reservation by the tagged SU succeeds at the first attempt. With probability $\alpha(1-\alpha)$, the channel reservation by the tagged SU succeeds after two reservation attempts, and so on. Associated with a particular $X$-cycle, as shown in Fig. $1, R$ denotes the number of reservation attempts until either the SU packet is discarded or transmitted. We have

$$
\begin{aligned}
E[R]= & 1-\alpha+2 \alpha(1-\alpha)+3 \alpha^{2}(1-\alpha)+\ldots \\
& +(M-1) \alpha^{M-2}(1-\alpha)+M \alpha^{M-1} \\
= & \sum_{m=0}^{M-1} \alpha^{m} .
\end{aligned}
$$

and

$$
\begin{aligned}
E[X]= & (1-\alpha)\left(b_{0}+K\right)+\alpha(1-\alpha)\left(b_{0}+b_{1}+K\right)+\ldots \\
& +\alpha^{M-1}(1-\alpha)\left(\sum_{m=0}^{M-1} b_{m}+K\right)+\ldots \\
& +\alpha^{M}\left(\sum_{m=0}^{M-1} b_{m}\right) \\
= & \sum_{m=0}^{M-1} \alpha^{m} b_{m}+\left(1-\alpha^{M}\right) K
\end{aligned}
$$

We thus obtain $\beta$ as given by

$$
\begin{aligned}
\beta & :=\frac{E[R]}{E[X]} \\
& =\frac{\sum_{m=0}^{M-1} \alpha^{m}}{\sum_{m=0}^{M-1} \alpha^{m} b_{m}+\left(1-\alpha^{M}\right) K}
\end{aligned}
$$

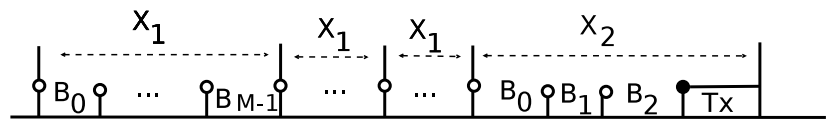

- Reservation Failure

Tx: Transmission

- Reservation Success

Fig. 1. Illustration of $X$ - cycle. Type $X_{1}$ is a cycle with packet discarded due to $M$ consecutive reservation failures. In contrast, type $X_{2}$ - cycle includes a transmission at a backoff stage $m\left(B_{m}\right)$.

\section{B. Derivation of $\alpha$}

Depending upon the tagged SU attempting a reservation on the common available channel in a chosen slot, the channel is either free from SUs activities with probability $P_{f}$ or occupied with probability $\left(1-P_{f}\right)$. By the law of total probability, it is easy to see that

$$
\begin{aligned}
P_{f} & =P_{f, f} P_{f}+P_{o, f}\left(1-P_{f}\right) \\
& =\frac{P_{f}}{1+P_{o, f}-P_{f, f}}
\end{aligned}
$$

where $P_{f, f}$ represents the conditional probability that the idle PU channel is free in the subsequent slot given that it is free in the current slot. Similarly, $P_{o, f}$ represents the conditional probability that the idle PU channel is free in the subsequent slot given that it occupied in the current slot.

1) Derivation of $P_{o, f}$ : It is known that a SU transmission lasts $K$ slots (in backoff unit time). Therefore, with probability $1 / K$, the SU transmissions ends in the last slot.

$P_{o, f}=\operatorname{Pr}($ next slot is free $\mid$ currently the last slot $) \times$

$\operatorname{Pr}$ (current slot is the last slot)

$+\operatorname{Pr}($ next slot is free $\mid$ currently not the last slot $) \times$

$\operatorname{Pr}$ (current slot is the last slot)

$$
=\frac{1}{K}
$$

2) Derivation of $P_{f, f}$ : Using the decoupling approximation, where all SUs attempt a channel reservation with probability $\beta$, the idle PU channel remains free for the subsequent slot when it is currently free if none of the collocated SUs starts a channel reservation process in the current slot. Clearly,

$$
P_{f, f}=(1-\beta)^{N}
$$

It follows from the above formulas that

$$
P_{f}=\frac{1}{1+K\left(1-(1-\beta)^{N}\right)}
$$

Finally, we obtain $\alpha$ as being

$$
\begin{aligned}
\alpha & :=1-P_{f} \\
& =\frac{K\left(1-(1-\beta)^{N}\right)}{1+K\left(1-(1-\beta)^{N}\right)}
\end{aligned}
$$




\section{Fixed Point Equation}

Following the decoupling approximation in [11],[7], we select $\beta$ to be the fixed point. In our analytical model, from the viewpoint of a tagged SU, we obtain $\beta$ while regarding the rest of collocated SUs as an environment. The resulting equation

$$
\beta=G(\alpha)
$$

can be interpreted as the SU's response equation. $\alpha$ captures the influence of the environment on the tagged SU, when each SUs uses the reservation attempt rate $\beta$. Thus $\alpha$ yields the coupling equation, i.e.,

$$
\alpha=H(N, \beta)
$$

The operating points $\alpha$ and $\beta$ of the channel reservation scheme are thus characterized by the solutions of the following fixed point equation:

$$
\begin{aligned}
\beta & =G(H(N, \beta)) \\
& :=\Pi(\beta)
\end{aligned}
$$

\section{Performance Analysis}

The parameters $P_{v}$ and $P_{\omega}$ complete the analysis about the channel reservation scheme. As illustrated in Fig. 1, every $X$ cycle ends with a SU transmission with probability

$$
P_{v}=(1-\alpha)^{M}
$$

Conditioned over the event that none of the $(N-1)$ remaining SUs should start the channel reservation procedure at the time when the tagged SU does, the conditional probability that a SU accesses the channel successfully is

$$
P_{v}=(1-\beta)^{N-1}
$$

We obtain the service time of a SU packet

$$
D s=\frac{1}{\beta(1-\beta)^{N-1}(1-\alpha)}
$$

and the aggregated throughput

$$
\Gamma=N \beta(1-\beta)^{N-1}(1-\alpha)
$$

By solving this equation, we obtain $\beta$ from which we further obtain $\alpha$.

\section{E. PU Collision Probability}

Under beacon-enabled mode, the distributed cognitive access operates as follows. A time-threshold policy with threshold $L$ is defined such that collective transmissions of SUs occur only when the following conditions are met: (i) the channel is sensed idle, (ii) $t<L$, in general $L$ is different from $L^{*}$ that maximizes the SU capacity. This mean that SU should transmit only when the time elapsed since the channel is idle, denoted $t$, is below a threshold $L$. By this, the access policy allows transmissions from SU only when the probability of colliding PU transmission is small.

With the channel reservation mechanisms enabled, this can be considered as a collision split sharing that splits the collision constraint, $\eta$, among SUs. Specifically, let $\sigma$ denotes a splitting coefficient, and be such, for a tagged SU, the equivalent collision limit is $\hat{\eta}=\sigma \eta$, with $\sigma \leq 1$. Given a threshold policy that enables the SU to access the channel only when the channel is idle and the condition $t<L$ is satisfied, a channel reservation mechanism can be incorporated into the particular threshold policy such that the collision constraint on the channel given by $\eta$ is collectively met.

It was established in [4] that the maximum capacity, denoted $J$, achievable on the channel is $J=\mu^{-1} \eta \rho(0)$, when the collision constraint is given by $\eta$ and the PU idle period is exponentially distributed. Conditioned over the event that none of the $(N-1)$ remaining SUs should start the channel reservation procedure at the time when the tagged SU does, here we can approximate the limit on individual time capacity achievable by the tagged SU to be $\hat{J}=\mu^{-1} \rho(0) \hat{\eta}$, which is given by

$$
\hat{J}=\frac{\eta \rho(0)(1-\beta)^{N-1}}{\mu}
$$

were $\sigma=(1-\beta)^{N-1}$.

\section{NUMERICAL RESULTS}

Numerical results are reported to illustrate the performance of the suggested policy for distributed cognitive access using some specific parameters of the system. The selection of the parameter values are not crucial but representative of the general behavior of the system. The number of collocated SUs that form the CR network varies from $N=3$ to $N=50$.

\section{A. Fixed point analysis}

In Fig. 2, we illustrate an example of the fixed point expressed in (18), as the rate $\beta$ at which every SUs of the network attempts a channel reservation. For a distributed network with $N$ the number of homogeneous collocated SUs, the SUs have the same configuration of backoff parameters. Each SU monitors the common available PU channel, and its backoffs behaves independently according to the channel reservation configuration. We compare the following channel reservation schemes: (i) a fixed scheme, marked "fixed" in the figure, where the number of backoff stage $M=2$, with mean backoff durations $b_{0}=4, b_{1}=8, b_{2}=16$; and (ii) an adaptive scheme, marked "adaptive" in the figure, where we remove the limit on the number of backoff stages allowed per SU packet (e.g., $M=7$ ) and set $b_{0}=4$. The average SU packet duration is fixed to $K=8$. The intersection with the line " $y=x$ " in equation (18) defines the fixed point. It is observed that for both fixed and adaptive backoff configurations, the reservation attempts rate of individual SUs remains almost constant after $N \geq 20$. It is also observed that the fixed point under fixed backoff configuration is always larger than the adaptive backoff configuration, which indicates that the 


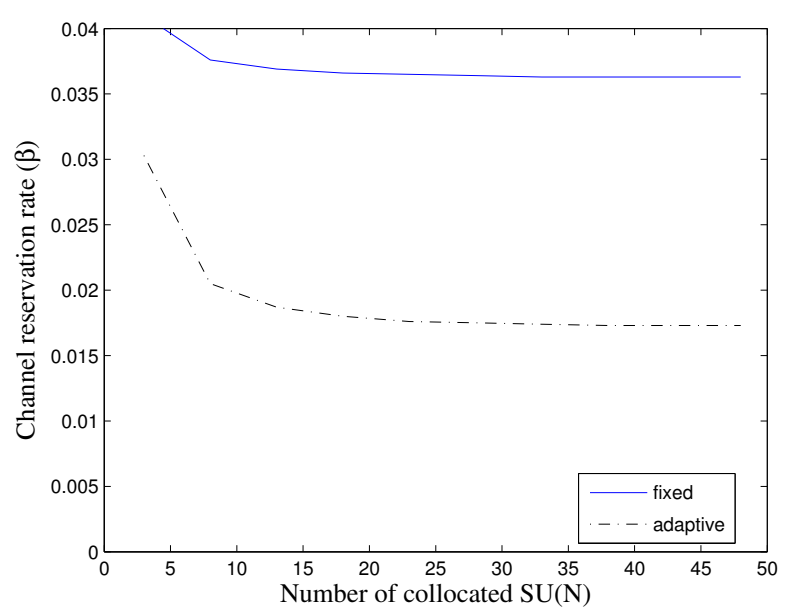

Fig. 2. Example of fixed point analysis

SU under adaptive backoff configuration attempts less often than under fixed configuration configuration. This is because larger $M$ allows the SU to expand their backoff duration more compared to the case where $M$ is fixed to a small value.

In Fig. 3 we illustrate the probability of a tagged SU finding a channel occupied right before it completes its backoff $\alpha$. It is observed that, when $N \leq 20$, the probability of a channel reservation failure is much larger when the tagged SU operates under fixed configuration compared to the adaptive configuration. This indicates that the probability to find a free channel increases. Consequently, when $N$ is small the probability to overlook some of the spectrum opportunities increases when SUs operate under adaptive configuration.

\section{B. Impact on the MAC performance}

Hereafter, we study the performance of the cognitive access under both the fixed and adaptive backoff configuration. In Fig. 4 we illustrate the aggregated throughput of the SUs network as a function of the number $N$ of collocated SUs. It is observed that, when the SUs operate under fixed backoff configuration, the resulting throughput decreases significantly with the number of collocated SUs compared to operation under adaptive backoff configuration. It is interesting to observe that, when $N \leq 15$, the performance with fixed configuration outperforms the one with adaptive configuration. But when $N>15$, the performance with adaptive configuration outperforms the one with fixed configuration. However, the performance gain increases with the number of collocated SUs.

In Fig. 5 we illustrate the service time for a SU packet under both the fixed and adaptive backoff setting as a function of the number of collocated SUs $N$. It is observed that, when $N \leq 15$ and SUs implemented the fixed backoff setting, the fixed setting achieves better average service time compared to the adaptive setting. In contrast, when $N>15$, the adaptive setting achieves better average service time compared to the fixed one. It is interesting to observe that the gap in the

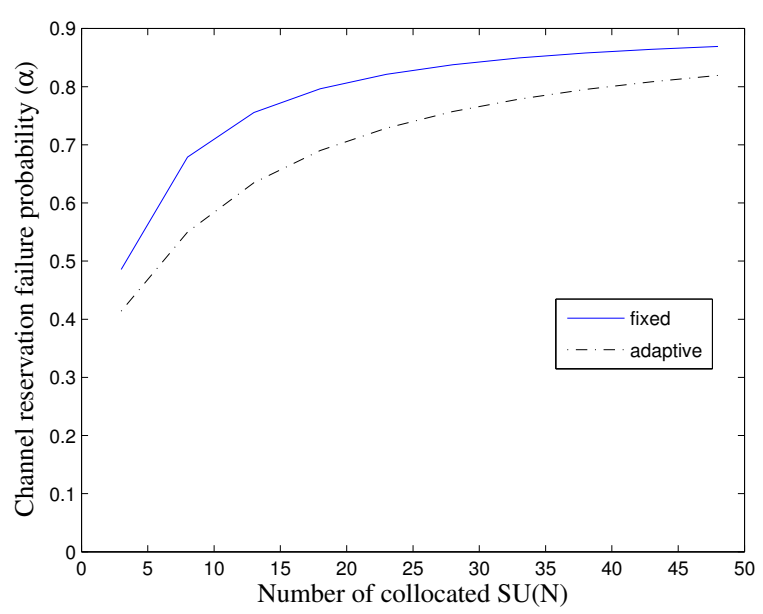

Fig. 3. Channel reservation failure probability

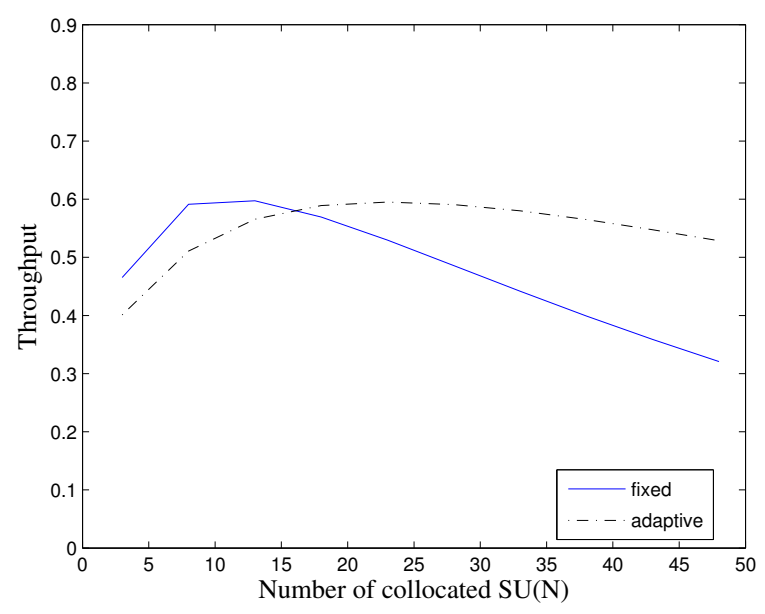

Fig. 4. Aggregated throughput for N collocated SUs

achievable average service time increases significantly with $N$.

\section{Impact on the time capacity}

In Fig. 6 we plot the maximum time capacity achievable when the SU adopts the adaptive backoff setting. We assume that the idle period is exponentially distributed with mean $\lambda^{-1}=150$, and the busy period is exponentially distributed with parameter denoted $\mu^{-1}=100$. The time capacity of the channel is $J=0.6$ when the collision constraint $\eta=0.01$. It is observed that, when $N=1$, the SU achieves the maximum capacity achievable on the channel. When $N>1$, however, its capacity decreases, which indicates how individual SU transmits less aggressively to accommodates other SUs. 


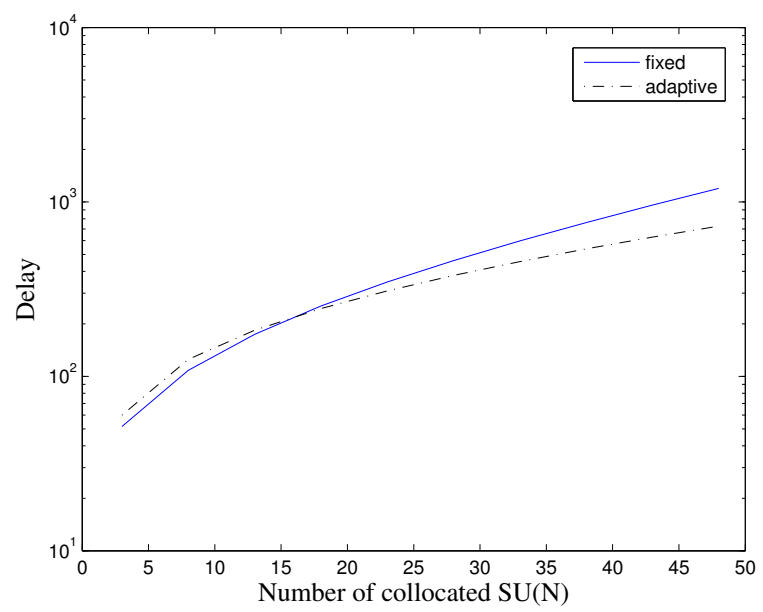

Fig. 5. Average service time of SU packet

\section{CONCLUSION}

We have introduced and analyzed a class of time-based threshold policy for distributed cognitive access, enabled by an out-of-band channel termed global common communication channel (CCC). Specifically, we have studied CSMA based coexistence mechanisms specific for collocated SUs, enabling them to transmit less aggressively to accommodate other SUs when sharing a common available channel. We have also reported an analytical framework that allows analysis of the suggested mechanism, the parameters of which can be obtained using fixed point techniques. The random access protocol reported in this paper characterizes how the SUs should search for transmission opportunities on the PU channel, coordinate among themselves to share available opportunities and transmit within the given collision constraint. The suggested model has been shown to enable characterization of individual SU under various backoff settings. Future work is on accuracy of the suggested model by using computer simulations.

\section{REFERENCES}

[1] Y. Chen, Q. Zhao, and A. Swami, "Joint design and separation principle for opportunistic spectrum access in the presence of sensing errors," IEEE Trans. Inf. Theory, vol. 54, no. 5, pp. 2053-2071, May 2008.

[2] S. Geirhofer, L. Tong, and B. Sadle, "Dynamic spectrum access in the time domain: Modeling and exploiting white space," IEEE Commun. Mag., vol. 45, no. 5, pp. 66-72, 2007.

[3] S. Huang, X. Liu, and Z. Ding, "Opportunistic spectrum access in cognitive radio networks," in Proc. IEEE INFOCOM '2008, Phoenix,AZ, Apr. 2008.

[4] — "Optimal transmission strategies for dynamic spectrum access in cognitive radio networks," IEEE Trans. Mobile Comput., vol. 8, no. 12 , pp. 1636 - 1648, Dec. 2009.

[5] S. C. Jha, M. Rashid, V. Bhargava, and C. Despins, "Medium access control in distributed cognitive radio networks," IEEE Trans. Wireless Commun., vol. 18, no. 4, pp. 41 - 51, Dec. 2011.

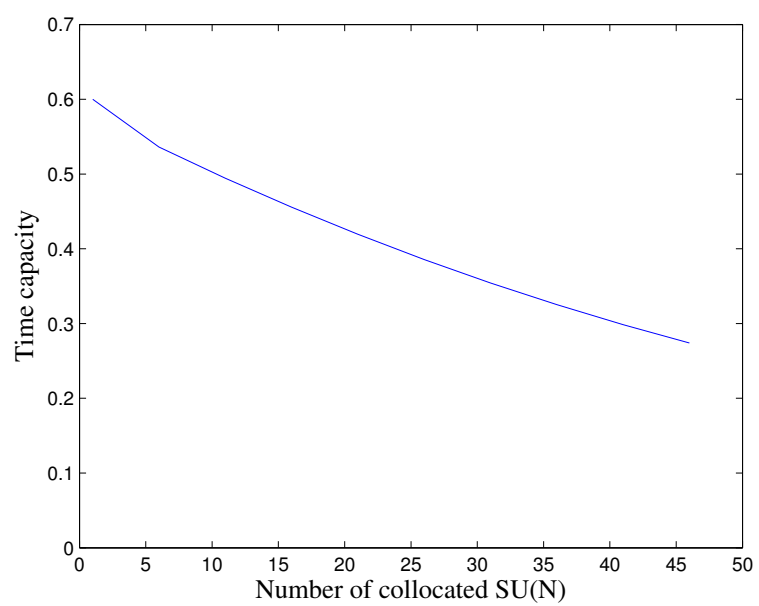

Fig. 6. Limit on individual time capacity achievable on the channel

[6] S. C. Jha, M. Rashid, U. Phuyal, and V. Bhargava, "Design of omcmac: An opportunistic multi-channel mac with qos provisioning for distributed cognitive radio networks," IEEE Trans. Wireless Commun., vol. 10 , no. 10 , pp. 3414 - 3425, Oct. 2011.

[7] A. Kumar, E. Altman, D. Miorandi, and M. Goyal, "New insights from a fixed point analysis of single cell ieee 802.11 wlans," IEEE/ACM Trans. on Networking, vol. 15, no. 3, pp. 1550 - 1561, Jun. 2007.

[8] X. Li, Q. Zhao, X. Guan, and L. Tong, "Optimal cognitive access of markovian channels under tight collision constraints," IEEE J. Sel. Areas Commun., vol. 29, no. 4, pp. 746-756, Mar. 2011.

[9] X. Ling, Y. Cheng, J. W. Mark, and X. Shen, "A renewal theory based analytical model for the contention access period of ieee 802.15.4 mac," IEEE Trans. Wireless Commun., vol. 7, no. 6, pp. 2340 - 2349, Jun. 2008.

[10] J. Mitola, "Cognitive radio technologies: An integrated agent for software defined radio," Ph.D. dissertation, KTH Royal Inst. of Technol., Stockhold, Sweden, 2000.

[11] C. K. Singh, A. Kumar, and P. M. Ameer, "Medium access control in distributed cognitive radio networks," ACM Wireless Networks, vol. 14 no. 4, Aug. 2008.

[12] B. Wang and K. R. Liu, "Advances in cognitive radio networks: A survey," IEEE J. Sel. Topics Signal Process., vol. 5, no. 1, pp. 5-23, Feb. 2011.

[13] Q. Xiao, Y. Li, X. Zhong, X. Xu, and J. Wang, "A unified approach to optimal opportunistic spectrum access under collision probability constraint in cognitive radio systems."

[14] S. Zhang, C. Zhu, J. K. O. Sin, and P. K. T. Mok, "A spectrum surveying framework for dynamic spectrum access networks," IEEE Trans. Veh. Technol., vol. 58, no. 8, pp. 4158-4168, Oct. 2009.

[15] Q. Zhao, S. Geirhofer, L. Tong, and B. Sadler, "Opportunistic spectrum access via periodic channel sensing," IEEE Trans. Signal Process. vol. 56, no. 2, pp. 785-796, Jan. 2008 